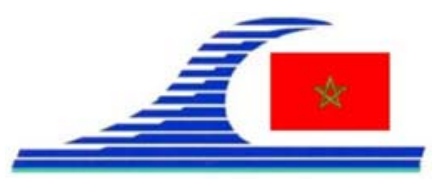

Conférence Méditerranéenne Côtière et Maritime

EDITION 2, TANGER, MAROC (2011)

Coastal and Maritime Mediterranean Conference

Disponible en ligne - http://www.paralia.fr - Available online

\title{
Contribution à l'étude hydrodynamique sédimentaire de la frange littorale de Zarzis (Tunisie)
}

\author{
Abdelfattah ATOUI ${ }^{1}$, Mouldi BRAHIM ${ }^{1}$, Chérif SAMMARI ${ }^{1}$ \\ 1. Institut National des Sciences et Technologies de la Me, 28 rue 2 mars 1934, \\ Salammbô, 2025 Tunis, Tunisie.abdelfattah.atoui@gmail.com
}

\section{Résumé :}

Pour élucider le transport sédimentaire qui prévaut dans la bande côtière située entre Ras Marmour et le port de Zarzis (partie sud du golfe de Gabès, Tunisie), des prélèvements de sédiment de surface $(\approx 0-20 \mathrm{~m})$ ont été réalisés. L'analyse granulométrique de ces échantillons a été faite en même temps que l'analyse d'une série temporelle de courant couvrant entre autres la campagne de collecte des échantillons.

La répartition de la taille moyenne de la fraction grossière des sédiments (> $63 \mu \mathrm{m})$ montre deux gradients bien marqués. Le premier concerne la différence entre la côte et le large alors que le second met en évidence des faciès complètement différents entre le Nord-Ouest et le Sud-Est. D'une manière plus explicite nous pouvons affirmer que la zone est marquée essentiellement par trois faciès sédimentaires : des sables grossiers, des sables moyens et des sables fins.

Les sables grossiers et moyens sont modérément classés alors que les sables fins sont mal classés. Les fonds qui sont couverts par des sables grossiers et des sables moyens ont subi une importante érosion qui est le signe de l'existence d'une dynamique assez importante. Au sud de la zone étudiée et au large, la dynamique près du fond est vraisemblablement moins importante comme en témoigne la couverture du fond marin par des sables fins.

Comme mentionné plus haut, nous avons obtenu et analysé une série temporelle de courant enregistré à 10 mètres de profondeur en face de Ras Marmour. Cette analyse a montré que la direction du courant moyen au cours de la période de mesure (29/10/2010 au 30/11/2010) est de secteur sud-est. Les modules de la vitesse varient entre 2 et $14 \mathrm{~cm} / \mathrm{s}$. En principe, cette dynamique génère des courants de dérive vers le nord-ouest et par conséquent le transport de sédiments s'effectue du sud-est vers le nord-ouest tout au moins pendant la période de la campagne. Or, le régime de houle qui prévaut dans la région est de direction est/nord-est et doit donc générer des courants de dérive du nordouest vers le sud-est. Et, c'est justement la principale observation déduite de notre analyse granulométrique. Il est alors légitime de penser que le régime de courant observé n'est pas compatible avec le courant moyen qui prévaut dans la région et constitue une situation exceptionnelle induite par des régimes particuliers de vents.

\section{Mots-clés :}

Courant - Granulométrie - Ras Marmour - Port de Zarzis 
La connaissance de la Mer :

un vecteur du développement durable en Méditerranée

\section{Introduction}

Le transport solide à un impact majeur économique et écologique sur les ouvrages maritime et le "by-pass" laguno-marin entre la lagune de Boughrara et la mer Méditerranée. Ce transport est l'effet observable des interactions entre plusieurs facteurs notamment les courants côtiers, l'évolution spectaculaire des constructions balnéaires et les coups du vent.

Un site reflétant ces effets, celle de la frange côtière de Zarzis situé au Sud-Est de la Tunisie entre $33^{\circ} 27^{\prime} 46^{\prime \prime}$ et $33^{\circ} 39^{\prime} 40^{\prime \prime}$ de latitude Nord et $11^{\circ} 10^{\prime} 29^{\prime \prime}$ et $11^{\circ} 13^{\prime} 23^{\prime \prime}$ de longitude Est (figure 1). Dans ce travail en s’intéresse à l'étude hydro-sédimentaire, en utilisant de donnés courantologique et granulométrique de la frange côtière de Zarzis, que s’intègre dans le cadre d'un projet national de gestion du littoral tunisien réalisé par le laboratoire du Milieu Marin de l’INSTM.

\section{Matériels et méthodes}

Deux campagnes d'échantillonnage des sédiments de surface ont été effectuées. La première a été effectuée au large, à bord du NO/ HANNIBAL le 24/07/2010, au niveau de l'isobathe $-20 \mathrm{~m}$, la deuxième a été effectuée à bord d'un Zodiac de l'INSTM pour prélever les sédiments dans les petits fonds entre 0 et 10 m le 29/10/2010 (figure 1).

Nous avons prélevés 24 échantillons des sédiments de surface entre 0 et $20 \mathrm{~m}$ de profondeur à l'aide d'une benne de type Van Veen. Les positions de prélèvements ont été effectuées par un GPS radar (Magellan explorist 100). L'analyse granulométrique a été effectuée sur une série de tamis de type AFNOR dont les mailes varient de 63 à $2000 \mu \mathrm{m}$.

Un Argonaut-MD/1.5 Mhz (Sonteck, USA) a été installé en face de Ras Marmour (N33³9.092'; E1102.946') durant la période allant du 29/10/2010 au 09/12/2010.

\section{Résultats et interprétation}

\subsection{Courant en face de Ras Marmour}

La vitesse du courant en face de Ras Marmour à une direction SE/NW variable entre 2 et $14 \mathrm{~cm} / \mathrm{s}$ (figures 2 et 3). Le diagramme du vecteur progressif montre un déplacement de la masse d'eau parallèlement à la côte de la zone d'étude avec quelque perturbation (figure 4). La polarisation de la vitesse constitue une situation exceptionnelle induite par des régimes particuliers, due à l'effet de la bathymétrie et de vents.

Le diagramme bâton de la figure 2 montre une évolution périodique dans le temps chaque 12 heures environ et ceci montre la dominance des harmoniques de la marée semi diurne $\mathrm{M}_{2}$ et $\mathrm{S}_{2}$ conformément aux résultats déduits à travers des travaux antérieurs tels que ceux de HATTOUR et al (2010), et de SAMMARI et al (2006). La figure 2 montre aussi des perturbations pendant les jours 20 et 28 Novembre et le 4 décembre. Cette perturbation est due à des agitations atmosphériques importantes. 
L'analyse en composante principale où EOFS de la vitesse horizontale montre que la vitesse suivant l'axe majeur est de $4.3 \mathrm{~cm} / \mathrm{s}$ et celle suivant l'axe mineur est de $1.8 \mathrm{~cm} / \mathrm{s}$. Le courant principal est de direction $129^{\circ}$ par rapport au Nord (figure 5).

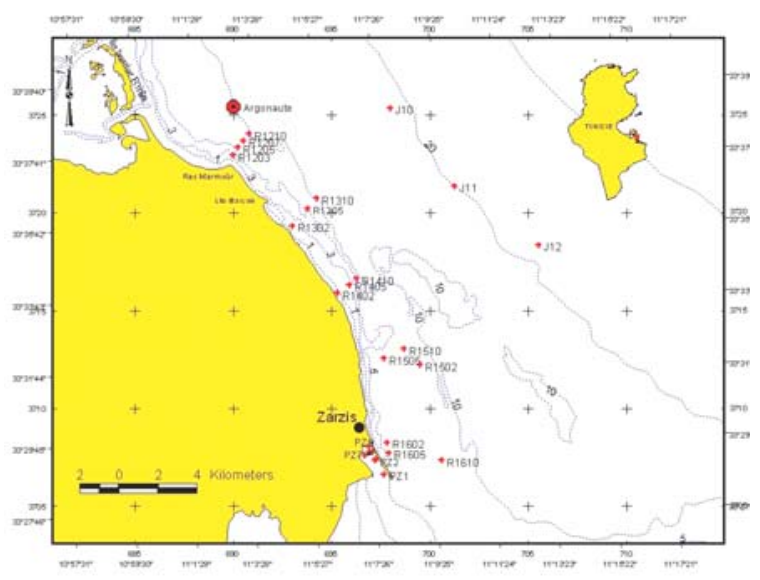

Figure 1. Stations de prélèvements des sédiments et position de l'Argonaute.

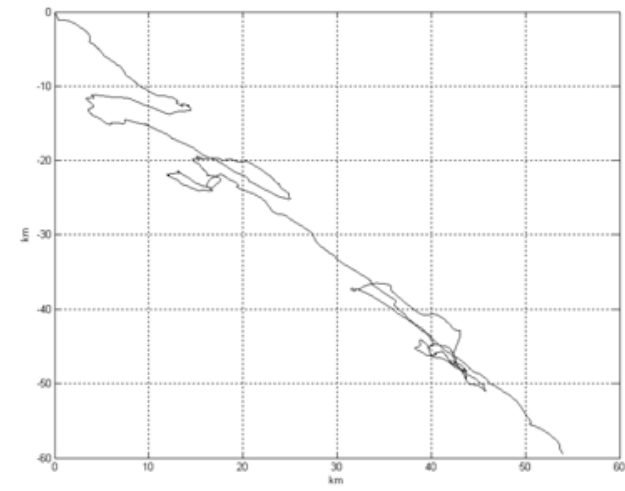

Figure 4. Diagramme du vecteur progressif de mouvement d'eau.

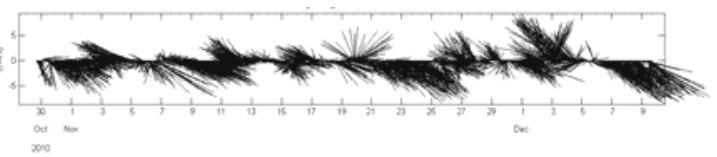

Figure 2. Le diagramme bâton de vecteur

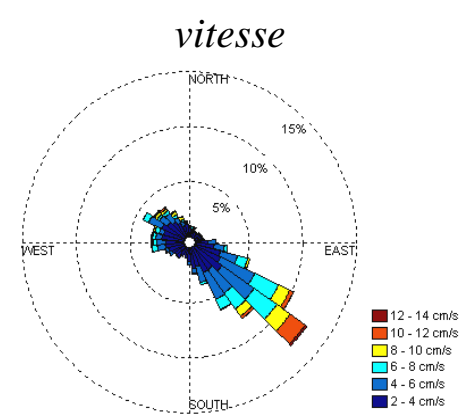

Figure 3. Rose de courant en face de Ras Marmour à $10 \mathrm{~m}$ de profondeur.

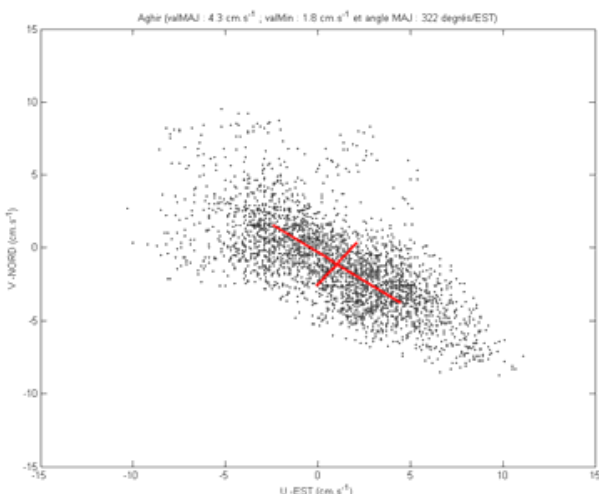

Figure 5. Analyse en EOF de vitesse horizontale.

\subsection{Répartition des grains des sédiments superficiels}

Suite à une analyse granulométrique, nous avons tracé les courbes cumulatives semilogarithmiques pour tous les échantillons et nous avons calculé la moyenne et l'écart type pour connaitre la nature et le classement des sédiments.

La figure 6 représente la répartition de la taille moyenne des grains de sable qui montre deux gradients assez importants. Un gradient est observé de la côte vers le large et un autre est du nord-ouest vers le sud-est. Ainsi la taille moyenne des grains de sable diminue de la côte vers le large et du nord-ouest vers le sud-est. Nous distinguons essentiellement trois faciès sédimentaire: des sable grossiers, des sables moyens et des 
La connaissance de la Mer :

un vecteur du développement durable en Méditerranée

sables fins (figure 6). Les sables grossiers et moyens sont modérément classés et les sables fins sont mal classés. Les fonds qui sont couverts par des sables grossiers et des sables moyens ont subit une érosion importante et la dynamique est assez importante. Au sud de la zone étudiée et au large, la dynamique est moins importante et le fond marin est couvert par des sables fins. La figure 7 met en évidence la circulation dominante qui est du nord-ouest vers le sud-est, responsable du transport des sédiments considérable du nord vers le sud.

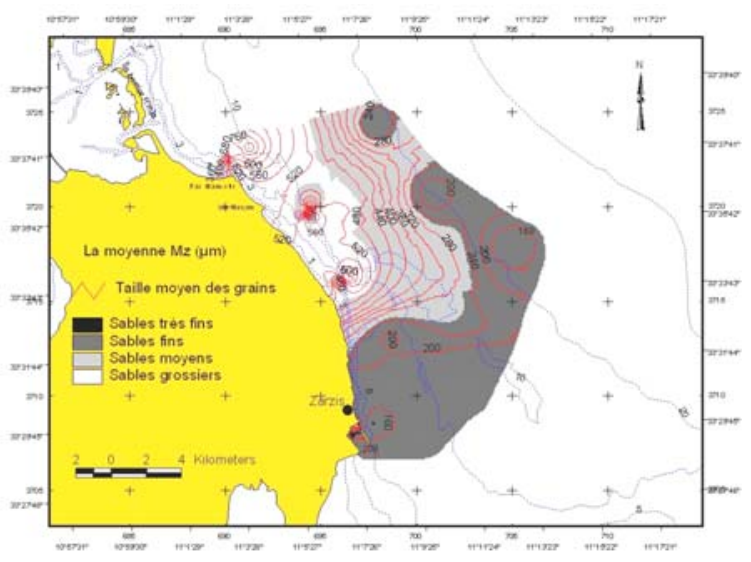

Figure 6. Evolution spatiale de la taille moyenne des échantillons du sable.

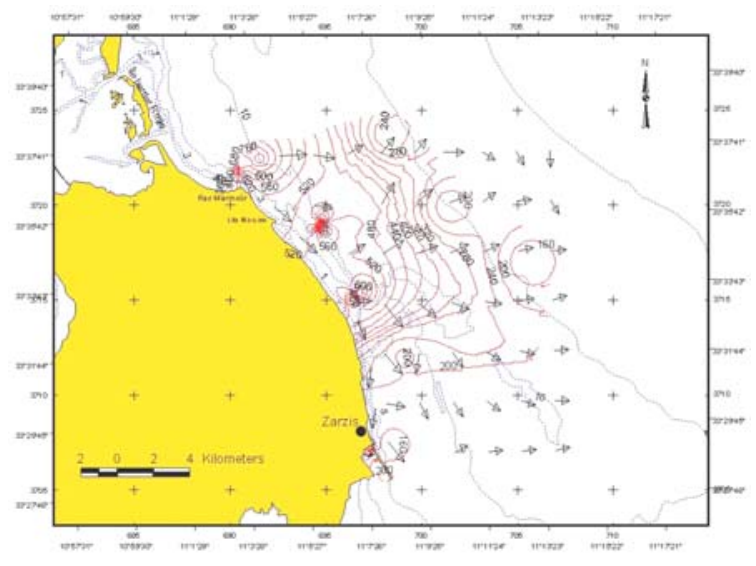

Figure 7. Courantologie à Zarzis par une approche sédimentologique.

\section{Conclusion}

La courantologie dans la frange littorale de Zarzis est due essentiellement à la marée semi-diurne dont le jusant est du Nord vers le sud et le flot est en sens contraire. Cette circulation est affectée au large par des perturbations atmosphériques suffisantes pour la naissance d'une houle de direction moyenne de Nord-Est.

La houle Nord-Est engendre des courants de dérive du nord-ouest vers le sud-est dans la frange littoral de Zarzis qu'est à l'origine d'un transport sédimentaire de Ras Marmour vers le port de Zarzis. Ceci est confirmé par l'accumulation des sédiments fins du côté du port de Zarzis et le tri granulométrique s'effectue du nord-vers le sud. Le fond marin entre 0 et $20 \mathrm{~m}$ de profondeur de cette zone est tapissé par trois faciès sableux de fins au grossiers.

\section{Références bibliographiques}

HATTOUR M.J., SAMMARI C., BEN NASSRALLAH S. (2010). Hydrodynamique du golfe de Gabès déduite à partir des observations de courants et de niveaux. Revue Paralia, $n^{\circ}$ 3, pp 3.1-3.12. doi:10.5150/revue-paralia.2010.003

SAMMARI C., KOUTITONSKY V.G., MOUSSA M. (2006). Sea level variability and tidal resonance in the Gulf of Gabès. Tunisia, Continental Shelf Research, 26, pp 338-350. doi:10.1016/j.csr.2005.11.006 